\title{
Chain Stability in Traffic Flow with Driver Reaction Delays
}

\author{
Rifat Sipahi Silviu-Iulian Niculescu
}

\begin{abstract}
A deterministic microscopic car-following model considering delayed reactions of human drivers is studied from systems perspective for chain stability of a configuration of vehicles arranged on a single lane cruising at constant velocity without changing lanes. Two different driving strategies accompanied by two different delay models are incorporated in this microscopic model. Arising analytical derivations from chain stability analysis help compare the effects of these strategies to and characterization of the traffic flow in the parameter space defining the delays and the coefficients representing the aggressiveness of the drivers. Illustrative examples are provided.
\end{abstract}

Index Terms - traffic dynamics, discrete delay, distributed delay, slinky effects, stability.

\section{Introduction AND PROBlem Statement}

According to a recent research note published by National Highway Traffic Safety Administration (NHTSA) [27], motor vehicle crashes were the leading cause for death in the US in 2002 for the ages between 3 and 33. This can only be seen as one of the many reasons why traffic behavior is a research focus since 1930s, [12]. As a consequence, numerous mathematical models have been developed, [6], [23], [7], [5], [28], [29], which characterize, according to their degree of detail, the traffic behavior considering single/multiple lanes, on/off ramps, traffic lights, roundabouts, etc.

Since the framework/ideas deployed to obtain mathematical models is too broad, we will focus on a particular subclass which is widely preferred [5], [12], [21], [20], [29] when studying traffic behavior: we consider a deterministic microscopic follow-the-leader type model in which drivers cruise at a constant velocity on a single-lane without changing lanes. Although this selection may simplify the model, it facilitates the understanding of the dynamics arising among the interaction of the vehicles under given driver behavior. Furthermore, such models enable linear and non-linear analytical development towards qualitative characterization of traffic flow [5], [7], [12], [14], [29] as well as multi-agent consensus/flocking problems [9], [19], [15], [22]. Besides the simplifications, however, the two crucial analysis that are needed to be performed on these models may become mathematically quite challenging. These analysis are (i) asymptotic stability (AS) [7], [5], [21], [20], [29] and (ii) chain stability (CS) [14], [17]. While stability will indicate exponential

RIFAT SIPAHI is with the Department of Mechanical and Industrial Engineering, Northeastern University, Boston, MA 02115. Email: rifatacoe.neu.edu

S.-I.NICULESCU is with the Laboratoire de Signaux et Systèmes (L2S), Supélec, 3, rue Joliot Curie, 91190, Gif-sur-Yvette, France. E-mail: Silviu.Niculescullss.supelec.fr
TABLE I

SOME EXAMPLES OF DELAY MEASURE. DELAYED REACTION OF HUMAN DRIVERS IN RESPONSE TO VARIOUS STIMULI

\begin{tabular}{cc}
\hline Measure of constant time delay, $\tau$ [sec] & Reference \\
\hline 0.496 & {$[4]$} \\
0.73 & {$[18]$} \\
0.7 & {$[8]$} \\
1.16 & {$[30]$} \\
1.13 & {$[1]$} \\
$0.75-1.0$ & {$[7]$} \\
$0.70,1.25$ & {$[10]$} \\
1.1 & {$[29]$} \\
\hline
\end{tabular}

decay of the response of the system states (velocity and position of vehicles) in time against impulsive perturbations; chain stability corresponds to attenuation of the periodic excitations among consecutive vehicles.

It is clear that the instability precludes any further analysis, thus chain stability is studied in the parameter space assuring asymptotic stability of the dynamics. When amplifications (attenuations) occur in frequency response, we will call the dynamics with (free of) 'slinky effects'. Clearly neither instability nor slinky effects are desirable for the traffic dynamics; and they should be carefully studied.

Presence of human drivers is still inevitable in traffic. As the central control decision-makers, human drivers close the feedback control loop in the traffic flow, human-in-the-loop dynamics [16]. Drivers can do multi-tasking and have rapid learning skills and anticipation capabilities, however their reactions are not instantaneous [5], [6], [7], [10], thus they add delays in decision-making and actuation, Table I. Delays mainly originate due to the time needed to receive a stimulus, become conscious, make decision and command actuation to the vehicle.

Delay in closed loop dynamics is a well-known source of poor performance (low damping) and robustness, and even instability, [17], [11]. In many cases, however, analyzing asymptotic stability and chain stability become non-trivial in presence of delays. Furthermore, since human behavior is not perfectly understood, it is still of question how to model the delayed reactions of the drivers. For instance, even for the case when delay $\tau$ is assumed constant, different numerical choices are possible, Table I. Consequently, delay modeling is another crucial component when studying traffic flow. In this paper, we deploy two different models inspired by the literature and authors' recent work: (a) discrete delay model, (b) distributed delay model. Discrete delay assumes that a 
decision made at time $t$ is based on the stimulus received at time $t-\tau$. On the other hand, drivers utilize their memory when they drive, [25], thus they receive stimuli continuously distributed over the history, prompting the need to model the received stimuli using distributed delays.

To our best knowledge, a complete analytical study considering various delay models and driving strategies with interconnection of AS and CS within the framework of traffic research has not been pursued in the open literature and we form our main objective along this line. Our aim is to develop analytical tools by using time delay systems approach to analyze chain stability of a deterministic microscopic traffic flow model. We adapt the two delay models for the driver behavior along with two different driving strategies utilized by these drivers. In brief, this paper combines the chain stability with asymptotic stability results in order to characterize the traffic behavior. It is of particular interest to derive simple analytical conditions in the parameter space of delay and driver aggressiveness coefficients, and to compare different driving strategies. We believe that research in this direction has impacts on understanding human behavior and its interconnection with AS/CS, studying automated car following control and semi-active driver assistance systems.

Notation. The notations are standard. Set of positive and negative real numbers are denoted by $\mathbb{R}_{+}$and $\mathbb{R}_{-}$, respectively. $\mathbb{C}_{+}$and $\mathbb{C}_{-}$represent the open right and left half of complex plane, respectively, whereas the imaginary axis is denoted by $j \mathbb{R}$, where $j=\sqrt{-1}$. We use $s$ for the Laplace variable.

The remaining part of the paper is organized as follows: In Section II, mathematical modeling of traffic flow and delays are presented. Section III presents the approach for the analysis of chain stability and the analytical tools are derived. Illustrative examples are given in Section IV. Section $\mathrm{V}$ concludes the paper.

\section{Mathematical Modeling}

\section{A. Traffic flow modeling without delays}

We consider a single-lane continuous-time deterministic microscopic car following model, in which a chain of vehicles travel at a constant velocity, the so-called quasi steady-state, without changing lanes, [6], [23], [12]. For more elaborate models, see the survey [12] and Intelligent Driver Model (IDM) in [29].

Strategy 1. Drivers create their decisions based on relative velocity between two consecutive vehicles. This suggests that the velocity difference is weighted $\alpha_{k}>0$ for decision making. Assuming vehicle dynamics as a double integrator, we have

$$
\ddot{x}_{k}(t)=\alpha_{k}\left(\dot{x}_{k+1}(t)-\dot{x}_{k}(t)\right),
$$

where $x_{k}$ is the velocity of the $k$ th vehicle, and the weighting $\alpha_{k}$ can be seen as proportional control gain or a measure of driver aggressiveness, see also Table II. We note that mathematical models similar to (1) also arise in traffic
TABLE II

NUMERICAL VALUES OF AGGRESSIVENESS COEFFICIENTS $\alpha_{k}$ AND $\mu_{k}$ DEPLOYED IN THE LITERATURE

\begin{tabular}{ccc}
\hline$\alpha_{k}$ & $\mu_{k}$ & Reference \\
\hline 3.0 & $2.0,2.8$ & {$[5]$} \\
2.0 & 2.0 & {$[7]$} \\
& 0.85 & {$[13]$} \\
& 1.0 & {$[14]$} \\
& {$[0,2]$} & {$[21]$} \\
& 0.37 & {$[31]$} \\
\hline
\end{tabular}

research [4], [5], [6], [12] and multi-agent consensus/flocking problems [9], [19], [15], [22].

Strategy 2. Drivers create their decisions based on the combination of relative position and relative velocity feedback. This strategy suggests that the velocity and position difference are used in decision making with the weighting factors $\alpha_{k}>0$ and $\mu_{k}>0$. Assuming again vehicle dynamics as a double integrator, we have

$$
\ddot{x}_{k}(t)=\alpha_{k}\left(x_{k+1}(t)-x_{k}(t)\right)+\mu_{k}\left(\dot{x}_{k+1}(t)-\dot{x}_{k}(t)\right),
$$

where similar arguments hold as in Strategy 1. Feedback of relative velocity and position can be seen as a proportionalderivative control to achieve consensus of $x_{k}(t) \rightarrow x_{k+1}(t)$ and $\dot{x}_{k}(t) \rightarrow \dot{x}_{k+1}(t)$ on the double integrator vehicle dynamics. The controller gains $\alpha_{k}$ and $\mu_{k}$ are measures of drivers' aggressiveness, Table II. See [5], [7], [12], [14], [29] for mathematical models similar to (2) arising in traffic flow and consensus/flocking of multi-agents [9], [19], [15], [22].

Remark 1: The governing dynamics for both Strategy 1 and Strategy 2 will exhibit a rigid body motion. Thus we expect to see, in Laplace domain, an $s=0$ pole of the velocity dynamics of each vehicle. This pole at $s=0$ does not play any role on the asymptotic stability, [25], thus when appropriate we will disregard it in the analysis. Such an approach can be seen as a way of analyzing AS and CS around constant velocity of the vehicles.

\section{B. Delay modeling}

1) Discrete delay model: One of the widely preferred delay model representing the delayed reactions of human drivers is 'discrete delay', $\tau>0$, which assumes that an action of a human driver at time $t$ is based on what is experienced at time $t-\tau$, [5], [7], [21], [20], [28], [29]. As a starting point, we shall follow the same assumption in this paper. For the case with slowly time varying delays, see for instance [24].

2) Distributed delay model: Motivated by authors' recent work, a different delay modeling can be seen attractive. It suggests that stimulus is received continuously over the history and it is retained in human memory and utilized for decision-making. Such an argument requires the implementation of distributed delays which can be seen as a continuous collection of discrete delays. Following these arguments, we 
utilize a uniform distribution $f(\tau)$ with which the memory of drivers is modeled,

$$
f(\tau)=\left\{\begin{array}{ccc}
1 / \delta & \text { if } & h<\tau<h+\delta \\
0 & \text { otherwise }
\end{array},\right.
$$

where $\delta>0$ is the memory size and $h \geq 0$ is the deadtime after which information in the memory can be utilized for a control action. Such a model is quite satisfactory for representing an average of the information available in the short-term memory as well as for introducing some heterogeneity among the drivers, [2], [3], [25]. Borrowing the entire asymptotic stability (AS) from authors' recent work [25], we will study chain stability perspective and present it along with AS results.

\section{Main Results: Slinky EfFects with Delays}

We wish to shortly explain chain stability and slinky effects. Assume that all vehicles are traveling at a constant velocity $\vartheta_{0}$ and the velocity of vehicle $k+1$ in (1) exhibits some periodic perturbations such that $\dot{x}_{k+1}(t)=\vartheta_{0}+v_{k+1} \sin (\omega t)$ where $v_{k+1}$ is the amplitude and $\omega$ is the frequency of this periodic function. Since the governing dynamics is linear, we shall remove the constant velocity, $\vartheta_{0}$, behavior of the vehicles and only consider the periodic perturbations. In this case, the response of the following vehicle ( $k^{t h}$ vehicle) in steady state (thus independent of $\vartheta_{0}$ ) will also be a periodic function in the form of $\dot{x}_{k}(t)=v_{k} \sin \left(\omega t+\phi_{k}\right)$ where $v_{k}$ is the amplitude of the periodic oscillations observed in the velocity of the $k^{t h}$ vehicle and $\phi_{k}$ is the relative phase angle between $\dot{x}_{k}(t)$ and $\dot{x}_{k+1}(t)$. Both $v_{k}$ and $\phi_{k}$ are frequency dependent. Slinky effects occur if the amplitudes of the velocity of the $k^{t h}$ vehicle become larger compared to that of $(k+1)^{s t}$ vehicle. In other words, if $\exists \omega \in \mathbb{R}$ for which $F(\omega)=v_{k}(\omega) / v_{k+1}(\omega)>1$ holds, then slinky effects occur. This indicates that perturbations upstream of the chain of vehicles will amplify from one vehicle to another, causing severe oscillation amplitudes. If such an undesired situation is avoided for $\forall k \in[1, n]$, and $\forall \omega \in \mathbb{R}$, then it will indicate chain stability.

The general idea of studying if slinky effects occur or not is to first obtain the transfer function between the two vehicles. For instance, without delays, Eq.(1) will yield a transfer function in Laplace domain, $h(s)=\frac{X_{k}(s)}{X_{k+1}(s)}=\frac{\alpha_{k} s}{s^{2}+\alpha_{k} s}$, where $X_{k+1}(s)$ and $X_{k}(s)$ are the Laplace transforms of $x_{k+1}(t)$ and $x_{k}(t)$, respectively. Using the transfer function, one can obtain $F(\omega)=|h(j \omega)|$ or alternatively $M(\omega)=$ $F(\omega)^{2}=|h(j \omega)|^{2}$ in order to avoid square root sign. By a frequency $(\omega)$ sweeping test, one can analyze if $\exists \omega \in \mathbb{R}$ for which $M(\omega)>1$ or not. As stated earlier, if $M\left(\omega^{*}\right)>1(<$ 1 ), this will indicate amplification (attenuation) of periodic perturbations at frequency $\omega^{*}$.

When delays are present in the dynamics, the analysis will become more complicated, as we shall see below. Furthermore, due to presence of delays, the dynamics will become more prone to exhibit slinky effects, which may not exist when delays are ignored. Following a similar idea, we will first obtain $M(\omega)$ function with delays and derive analytical conditions with which chain stability can be characterized.

\section{A. Case 1. Strategy 1 with discrete delay}

Let us consider that decisions made by the drivers (right hand side of (1)) are based on what was experienced earlier. For this, the dynamics needs to be re-written considering delays: we assume that it takes $\tau_{k}$ and $\tau_{k+1}$ amount of time for the driver $k$ to anticipate the velocity of his vehicle and the preceding one, respectively. In order to maintain the formulation general, we state that $\tau_{k}$ and $\tau_{k+1}$ are not necessarily identical. This modifies (1) as

$$
\ddot{x}_{k}(t)=\alpha_{k}\left(\dot{x}_{k+1}\left(t-\tau_{k+1}\right)-\dot{x}_{k}\left(t-\tau_{k}\right)\right),
$$

from which we obtain the transfer function $h\left(s, \tau_{k}, \tau_{k+1}\right)$ after canceling out $s=0$ as per Remark 1 ,

$$
h\left(s, \tau_{k}, \tau_{k+1}\right)=\frac{\alpha_{k} e^{-\tau_{k+1} s} s}{s^{2}+\alpha_{k} e^{-\tau_{k} s} s} .
$$

When delays are zero, $\left|h\left(j \omega, \tau_{k}, \tau_{k+1}\right)\right| \leq 1$ holds for $\forall \omega \in \mathbb{R}$, but with delays different behavior is expected. The function $M(\omega)=\left|h\left(j \omega, \tau_{k}, \tau_{k+1}\right)\right|^{2}$ becomes

$$
M(\omega)=\frac{\alpha_{k}^{2}}{\alpha_{k}^{2}+\omega^{2}-2 \omega \alpha_{k} \sin \left(\beta_{k}\right)},
$$

where $\beta_{k}=\tau_{k} \omega \neq 0$. We now seek conditions in the parameter space of $\tau_{k}$ versus $\alpha_{k}$ for which $M(\omega)>1$, $\exists \omega \in \mathbb{R}$. Such a case is possible only when

$$
0<\omega<2 \alpha_{k} \sin \left(\tau_{k} \omega\right)
$$

holds. Consequently, for slinky effects it is necessary that $2 \alpha_{k} \sin \left(\tau_{k} \omega\right)>0$, which is satisfied when $0<\omega<\pi / \tau_{k}$.

Let us re-write the inequality (7) as

$$
\frac{1}{2 \alpha_{k} \tau_{k}}<\frac{\sin \left(\tau_{k} \omega\right)}{\tau_{k} \omega}
$$

Notice that, right hand side of the above inequality is always upper bounded by 1 . Thus, it is necessary and sufficient that $\alpha_{k} \tau_{k}>1 / 2$ holds such that the case $M(\omega)>1, \exists \omega \in \mathbb{R}$ is possible. The boundary of this condition depicts nothing but a hyperbola on the first quadrant of $\tau_{k}$ versus $\alpha_{k}$ plane, above which 'slinky effects' are present, Fig 1. It is critical to state that slinky effects do not claim anything on the stability of the dynamics, which should be separately analyzed. Since this is not within the scope of this paper, we give the stability condition in the following:

Stability condition of (4), [17]. Dynamics governed by the delay differential equation in (4) is stable if and only if $\tau_{k} \alpha_{k}<\frac{\pi}{2}$.

The boundary of the stability condition interestingly turns out to be another hyperbola, which is $\pi$ times larger than the one found for slinky effects. Parametric combination of $\left(\tau_{k}, \alpha_{k}\right)$ above and below this hyperbola indicates instability and stability, respectively, while any $\left(\tau_{k}, \alpha_{k}\right)$ pair on the hyperbola will create a harmonically oscillating (marginally 
stable) dynamics. Figure 1 summarizes the above calculations and discussions, on which three regions are indicated based on the slinky effects and the asymptotic stability of (4). Finally, one can also derive a conservative lower bound $\underline{\omega}$ where the inequality (7) is always violated for $\omega>\underline{\omega}$. This bound can be easily computed as $\underline{\omega}=2 \alpha_{k}$.

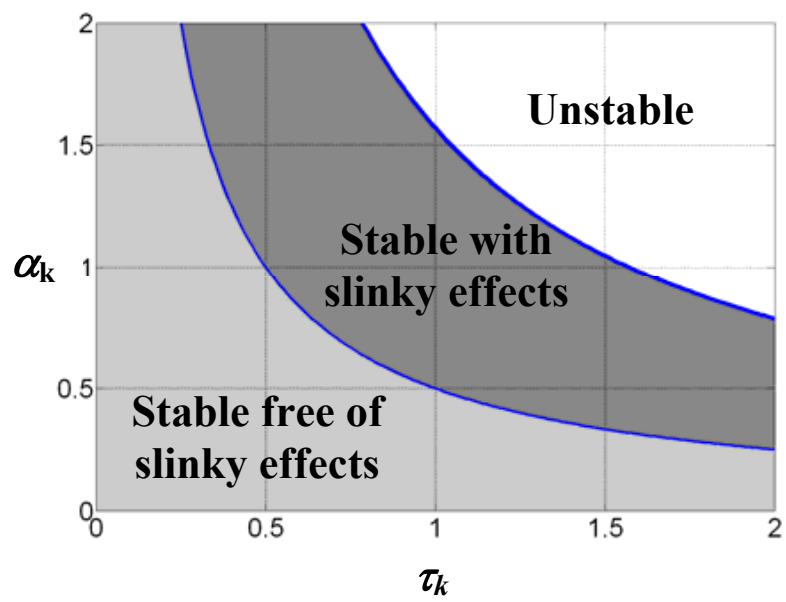

Fig. 1. Characterization of traffic flow dynamics in (4) with respect to stability and slinky effects in delay versus driver aggressiveness.

\section{B. Case 2. Strategy 2 with discrete delay}

In this case, it is assumed that decisions of the drivers are not only based on the relative velocity, but also on the headway (relative position). Thus, the governing dynamics with this driving strategy along with delays will be in the following form

$$
\begin{array}{r}
\ddot{x}_{k}(t)=\mu_{k}\left(x_{k+1}\left(t-\tau_{k+1}\right)-x_{k}\left(t-\tau_{k}\right)\right) \\
+\alpha_{k}\left(\dot{x}_{k+1}\left(t-\tau_{k+1}\right)-\dot{x}_{k}\left(t-\tau_{k}\right)\right)
\end{array}
$$

Similar to the previous case, we wish to analyze in the frequency domain $\omega$ the function $M(\omega)$, which is found as

$$
M(\omega)=\frac{\rho(\omega)}{\omega^{4}-2 \omega^{3} \alpha_{k} \sin \left(\beta_{k}\right)-2 \omega^{2} \mu_{k} \cos \left(\beta_{k}\right)+\rho(\omega)},
$$

where $\rho(\omega)=\mu_{k}^{2}+\omega^{2} \alpha_{k}^{2}$ and $\beta_{k}=\tau_{k} \omega \neq 0$. For chain stability, we need $M(\omega)<1$ for $\forall \omega \in \mathbb{R}$. Let us investigate if this can be assured by some parametric choice of $\alpha_{k}, \mu_{k}$ and $\tau_{k}$. If $M(\omega)<1$, then $g(\omega)=\omega^{2}-2 \omega \alpha_{k} \sin \left(\beta_{k}\right)-$ $2 \mu_{k} \cos \left(\beta_{k}\right)>0$ should hold, for $\forall \omega \in \mathbb{R}-\{0\}$. For this, it is sufficient that $\Delta=\alpha_{k}^{2} \sin ^{2}\left(\beta_{k}\right)+2 \mu_{k} \cos \left(\beta_{k}\right)<0$.

Scenario 1. Given any $\alpha_{k} \neq 0$ and $\mu_{k} \neq 0$, it is sufficient that $\beta_{k} \in(-\pi / 2,0),(\bmod 2 \pi)$, for $\Delta>0$ to hold. This leads to two real $\omega$ solutions of $g(\omega)=0, \omega_{1}$ and $\omega_{2}$, that are with opposite signs. Assuming $\omega_{1}>0$, dynamics will be free of slinky effects for any $\omega$ larger than $\omega_{1}$, while such effects will occur for $0 \leq \omega \leq \omega_{1}$ only.

Scenario 2. If $\beta_{k} \in(\pi / 2,3 \pi / 2),(\bmod 2 \pi)$, then (a) $\Delta>$ 0 which is similar to Scenario 1 ; (b) $\Delta=0$ and dynamics is free of slinky effects for $\forall \omega-\left\{\omega^{*}\right\}$, where $\omega^{*}=\alpha_{k} \sin \left(\beta_{k}\right)$; (c) $\Delta<0$ and dynamics is free of slinky effects for $\forall \omega$.

Finally, notice that one can derive a conservative lower bound $\underline{\omega}$ where $g(\omega)>0$ is always satisfied for $\omega>\underline{\omega}$. This lower bound can be found by replacing in a conservative sense $\sin \left(\beta_{k}\right)=1$ and $\cos \left(\beta_{k}\right)=1$ and choosing the positive $\omega$ solution, which is becomes $\underline{\omega}=\alpha_{k}^{2}+\sqrt{\alpha_{k}^{2}+2 \mu_{k}}$.

Remark 2: Recall that $\beta_{k}=\tau_{k} \omega$ and $\omega \in \mathbb{R}$. Hence, for any given delay $\tau_{k}$, one can choose $\omega$ to create any of the two scenarios listed above. In conclusion, we state that chain stability is not possible, except slinky effects do not occur in some intervals of $\omega$ and when $\omega>\underline{\omega}$. These properties distinguish this strategy with respect to Strategy 1 .

Stability condition of (9), [17]. We state that stability of dynamics in (9) should accompany the chain stability analysis presented above. This system is stable for $0 \leq \tau_{k}<\tau_{k}^{*}$ where $\tau_{k}^{*}=\frac{1}{\omega_{k}^{*}}\left(\tan ^{-1}\left(\frac{\alpha_{k} \omega_{k}^{*}}{\mu_{k}}\right) \mp 2 \pi \ell\right), \omega_{k}^{*}=\sqrt{\frac{\alpha_{k}^{2}+\sqrt{\alpha_{k}^{4}+4 \mu_{k}^{2}}}{2}}$ and $\ell$ is a counter guaranteeing the minimum positive $\tau_{k}^{*}$.

\section{Case 3. Strategy 1 with distributed delay}

We implement the memory effects on the governing dynamics formed by Strategy 1. For this, we utilize the uniform distribution presented earlier in (3). Consequently, the generalized dynamics becomes

$$
\ddot{x}_{k}(t)=\alpha_{k} \int_{0}^{\infty} f(\tau)\left(\dot{x}_{k+1}(t-\tau)-\dot{x}_{k}(t-\tau)\right) d \tau .
$$

In principle, Eq. (11) defines a traffic dynamics in which drivers perform their decisions based on what they continuously observed in the history during a memory window with finite size $\delta \neq 0$, and the information in the memory is retained and used in the decision-making process. Notice that the choice of a Dirac distribution for $f(\tau)$, i.e. $\delta \rightarrow 0$, will resume the discrete delay model studied in the previous subsection.

Similar to previous cases, we first obtain the transfer function from (11). This can be easily done by noticing that the right hand side of this equation is a convolution integral. Canceling $s=0$ as per Remark 1, we obtain

$$
\frac{X_{k}(s)}{X_{k+1}(s)}=\frac{\alpha_{k} F(s)}{s+\alpha_{k} F(s)}=\frac{\alpha_{k} e^{-h s}\left(1-e^{-\delta s}\right)}{\delta s^{2}+\alpha_{k} e^{-h s}\left(1-e^{-\delta s}\right)}
$$

which is used to derive $M(\omega)$ function,

$$
M(\omega)=\frac{\chi(\omega)}{\delta^{2} \omega^{4}-8 \omega^{2} \delta \alpha_{k} \sin ^{2}(\delta \omega / 2) \cos (\phi)+\chi(\omega)}
$$

where $\chi(\omega)=16 \alpha_{k}^{2} \sin ^{4}(\delta \omega / 2), \phi=\pi / 2-\delta \omega / 2-h \omega$. Furthermore, the mean of the uniform distribution is given by $m=h+\delta \omega / 2$, hence $\phi=\pi / 2-m \omega$. Recall that we need $M(\omega)<1, \forall \omega \in \mathbb{R}$, for chain stability. In the sequel, we investigate if this can be possible for some parametric choice defining the uniform memory. For $M(\omega)<1$, the following inequality should be satisfied, assuming $\omega \neq 0$,

$$
\delta^{2} \omega^{2}-8 \delta \alpha_{k} \sin ^{2}(\delta \omega / 2) \sin (m \omega)>0
$$


Further manipulations yield the following condition

$$
\frac{1}{2 \delta \alpha_{k}}>\left(\frac{\sin (\delta \omega / 2)}{\delta \omega / 2}\right)^{2} \sin (m \omega)
$$

Notice that $0<\sin ^{2}(x) / x^{2}<1$, thus the above condition holds for $\forall \omega \in \mathbb{R}$ if $\delta \alpha_{k}<1 / 2$. This is obviously a conservative condition, but it gives a relationship between the memory size and driver aggressiveness guaranteeing chain stability without any dependency on the mean delay, $m=h+\delta / 2$ and ultimately the deadtime $h$. Similar to the previous case, one can obtain a lower bound $\underline{\omega}$ on $\omega$, such that the inequality (14) always holds for any $\omega>\underline{\omega}$, and ultimately the dynamics is free of slinky effects. This lower bound is given by $\underline{\omega}=2 \sqrt{2 \alpha_{k} / \delta}$. We conclude this section by directing interested readers to [25] for asymptotic stability analysis of (11).

\section{ILLUSTRATIVE EXAMPLES}

\section{A. Case 1}

Let us illustrate three different frequency response behavior; first one corresponds to stable dynamics free of slinky effects $\alpha_{k}=0.5, \tau_{k}=0.75$, second one is stable but exhibits slinky effects $\alpha_{k}=0.7, \tau_{k}=1.0$ while the third case is chosen at the boundary $\alpha_{k}=0.5, \tau_{k}=1.0$ where stable dynamics transits from 'chain stable' to 'slinky effects', i.e., $\tau_{k} \alpha_{k}=1 / 2$. In Fig 2, the frequency response is depicted. As expected, the choice of $\left(\alpha_{k} \tau_{k}\right)$ comply with the analytical developments and Fig 1.

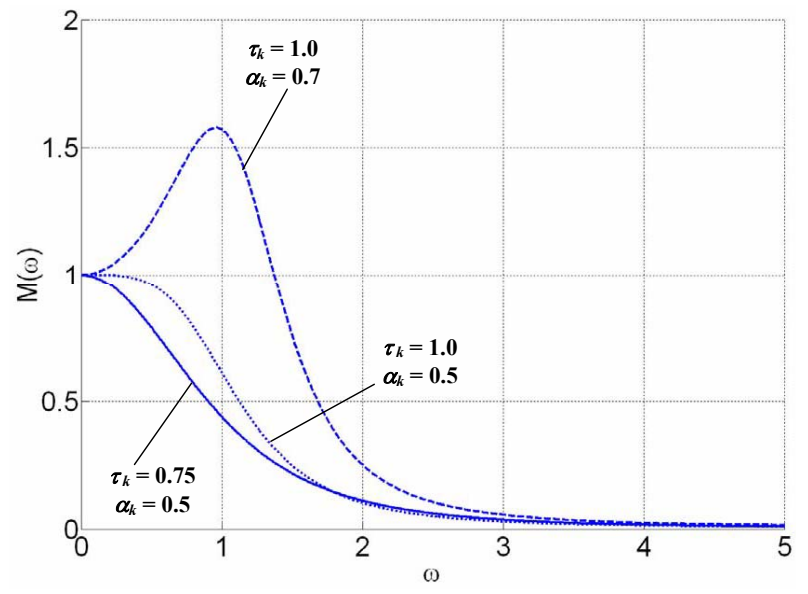

Fig. 2. Frequency response plot, $M(\omega)$ in Eq.(6) versus $\omega$ with $\tau_{k}, \alpha_{k}$ chosen from Fig 1.

\section{B. Case 2}

Let us now choose $\tau_{k}=0.1$ and obtain the variation of $M(\omega)$ function with respect to two driver aggressiveness coefficients $\alpha_{k}$ and $\mu_{k}$ and frequency domain $\omega$. The coefficients are chosen in the range of $[1,2.5]$, while to reduce the number of variables to depict a 3-dimensional view, $\omega$-axis is dropped, and instead, maximum of $M(\omega)$ along this axis is computed for each choice of $\alpha_{k}$ and $\mu_{k}$. Since this maximum is the indicator of slinky effects (if greater than 1), no information is lost in the analysis. Furthermore, results are presented in logarithmic scale in Fig 3 in order to obtain a convenient color gradient. Notice that, for chain stability it is required that $\log (\max (M(\omega)))<0$, however, in the parameter space $\left(\alpha_{k}, \mu_{k}\right)$ chosen, this does not occur. Next, we perform the stability analysis of the dynamics and obtain the maximum delay $\tau^{*}$ below which the dynamics is stable. This result is then converted to iso- $\tau^{*}$ contours that are superposed on top of $\log (\max (M(\omega)))$-surface. Notice that iso- $\tau^{*}$ contours range from 0.7 to 0.385 , thus our choice above, $\tau_{k}=0.1$, does not violate the stability on the chosen $\left(\alpha_{k}, \mu_{k}\right)$ plane.

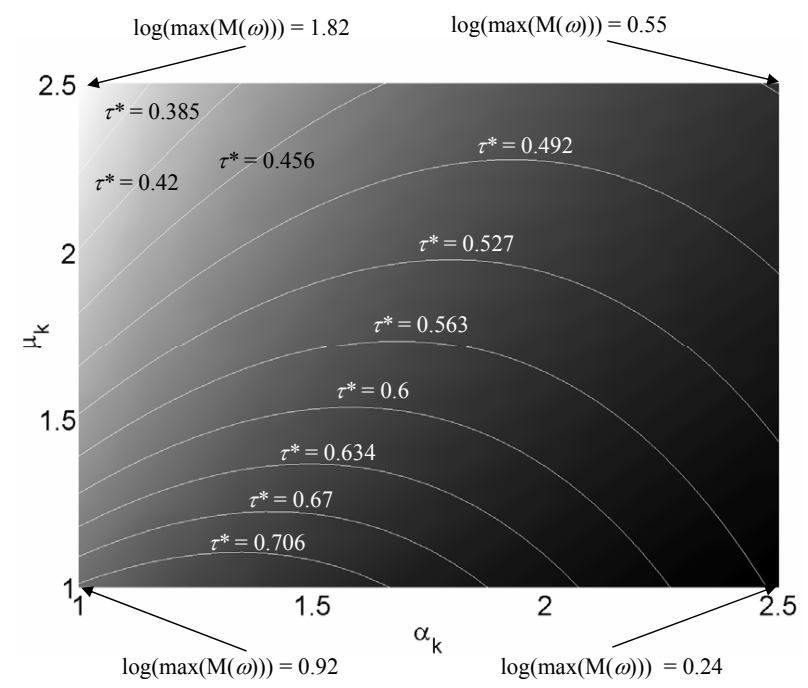

Fig. 3. Frequency response plot (gray sale), $\log (\max (M(\omega)))$ for $\tau_{k}=0.1$ versus $\alpha_{k}$ and $\mu_{k}$, superposed with contours indicating the iso maximum allowable delay, $\tau^{*}$ curves.

\section{Case 3}

Choosing driver aggressiveness $\alpha_{k}=2.0$, we follow a similar methodology as in Case 2, where we compute the maximum of $M(\omega)$ function by frequency sweeping. These maximum points are mapped on $\delta$ and $h$ parameters of the uniform distribution, while in parallel analyzing the stability of the dynamics in $(h, \delta)$ parameter space, [25]. Results are depicted in Fig 4, where shaded regions correspond to asymptotically stable dynamics. These regions are divided into two, based on $\max (M(\omega))$. If $\max (M(\omega))<1$, we label those regions as 'stable, no slinky effects', whereas $\max (M(\omega)) \geq 1$ indicates 'stable, with slinky effects'. Remaining part of the parameter space is not of interest since it corresponds to unstable dynamics.

Recall that we derived a conservative condition between $\alpha_{k}$ and $\delta$. If $\alpha_{k} \delta<1 / 2$, it is guaranteed that the dynamics is free of slinky effects. Consequently, for this case study, $\delta<$ $1 / 4$ guarantees slinky free dynamics. This conservative upper bound can easily be verified with the results obtained from 
the numerical computations, Fig 3. The boundary between the two gray regions is above the level $\delta=1 / 4$.

Remark 3: Notice that for a fixed $\alpha_{k}$ in Case 1, the delay interval allowable without creating slinky effects is about $31 \%$ of the entire stability interval $0 \leq \tau_{k}<\tau_{k}^{*}$. While chain stability is impossible to obtain in Case 2, parameter space corresponding to chain stability is about $18 \%$ of the entire shaded area for Case 3, Fig 4.

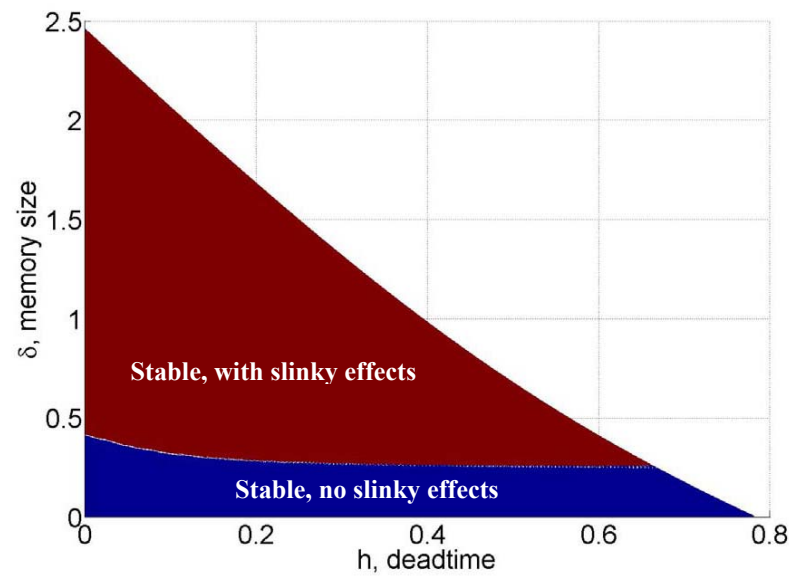

Fig. 4. Characterization of slinky effects with respect to $h$, deadtime and $\delta$, memory size.

\section{CONCLUSIONS}

Over a linear deterministic microscopic follow-the-leader type model, chain stability conditions are analytically derived for various driving strategies including delayed reactions of human drivers. It is shown that both delays in the reactions of human drivers and strategies the drivers choose for following their leaders become sources of loosing the chain stability. Particularly, the study is performed in the parameter space of the delays and the coefficients representing the aggressiveness of the drivers. Illustrative examples support the derived analytical results. It is foreseen that research along the same line has impacts on understanding chain stability of traffic flow as well as consensus problems in which inevitable delays contaminate decision-making.

\section{REFERENCES}

[1] H. Alm, L. Nilsson, "Changes in driver behaviour as a function of handsfree mobile phones: A simulator study", Accident Analysis and Prevention, 26, 441451, 1994.

[2] F.M. Atay, "Delayed-feedback control of oscillations in non-linear planar systems", Int. J. Control, 75, 297-304, 2002.

[3] F.M. Atay, "Distributed delays facilitate amplitude death of coupled oscillators", Phys. Rev. Lett., 91(9), 094101, 2003.

[4] B. Greenshields, "Reaction time in automobile driving", Journal of Applied Psychology, 20, 353357, 1936.

[5] Bando, M. Hasebe, K. Nakanishi, A. Nakayama, "Analysis of Optimal Velocity Model with Explicit Delay," Physical Review E, 58, 54295435, 1998.

[6] R.E. Chandler, R. Herman, E.W. Montroll, "Traffic Dynamics: Analysis of Stability in Car Following," Operational Research, 7(1), 165184, 1958.
[7] L.C. Davis, "Modifications of the Optimal Velocity Traffic Model to Include Delay Due to Driver Reaction Time," Physica A, 319, 557567, 2003.

[8] E. Dureman, C. Boden, "Fatigue in simulated car driving. Ergonomics", 15, 299308, 1972.

[9] L. Fang, P.J. Antsaklis, "Information Consensus of Asynchronous Discrete-Time Multi-Agent Systems", American Control Conference, Portland, OR, 2005.

[10] M. Green, “'"How Long Does It Take to Stop?" Methodological Analysis of Driver Perception-Brake Times," Transportation Human Factors, 2, 195-216, 2000.

[11] K. Gu, V.L. Kharitonov, J. Chen, Stability of Time-Delay Systems. Birkhauser: Boston, 2003.

[12] D. Helbing, Traffic and Related Self-Driven Many-Particle Systems, Reviews of Modern Physics, 73, 1067-1141, 2001.

[13] D. Helbing, B. Tilch, "Generalized Force Model of Traffic Dynamics," Physical Review E, 58, 133-138, 1998.

[14] K. Konishi, H. Kokame, K. Hirata, "Decentralized Delayed-Feedback Control of an Optimal Velocity Traffic Model," The European Physical Journal B, 15, 715-722, 2000.

[15] D. Lee, M W. Spong, 2006, "Agreement with Non-Uniform Information Delays", American Control Conference, Minneapolis, MN.

[16] R.M. Murray, Control in an Information Rich World: Report of the Panel on Future Directions in Control, Dynamics, and Systems, Society for Industrial \& Applied Mathematics, Philadelphia, PA, July 2003.

[17] S.-I. Niculescu, Delay Effects on Stability: A Robust Control Approach. Springer-Verlag: Heidelberg, LNCIS, 269, 2001.

[18] O. Norman, "Braking distance of vehicles from high speed. Public Roads", 27, 159169, 1952.

[19] R. Olfati-Saber, R.M. Murray, "Consensus Problems in Networks of Agents with Switching Topology and Time-Delays", IEEE Transactions on Automatic Control, 49(9), 1520-1533, 2004.

[20] G. Orosz, G. Stepan, "Hopf bifurcation calculations in delayed systems with translational symmetry", Journal of Nonlinear Science, 14(6), 505-528, 2004.

[21] G. Orosz, R.E. Wilson, B., Krauskopf, "Global Bifurcation Investigation of an Optimal Velocity Traffic Model with Driver Reaction Time," Physical Review E, 70(2), Art. No. 026207 Part 2, 2004.

[22] W. Ren, R.W. Beard, E.M. Atkins, "A Survey of Consensus Problems in Multi-agent Coordination", American Control Conference, Portland, OR, 2005.

[23] R.W. Rothery, "Transportation Research Board (Trb) Special Report 165," in Traffic Flow Theory, 2nd Edition, N. H. Gartner, C. J. Messner, and A. J. Rathi, Eds., 1998.

[24] R. Sipahi, S.-I., Niculescu, "Slow Time-Varying Delay Effects - Robust Stability Characterization of Deterministic Car Following Models", IEEE International Conference on Control Applications, October 2006, Munich, Germany.

[25] R. Sipahi, F.M. Atay, S.-I. Niculescu, Stability of Traffic Flow with Distributed Delays Modeling the Memory Effects of the Drivers, SIAM Applied Mathematics, 68(3), 738-759, 2007.

[26] G. Stepan, Retarded Dynamical Systems: Stability and Characteristic Function, New York: Longman Scientific \& Technical, co-publisher John Wiley \& Sons Inc., US., 1989.

[27] R. Subramanian, "Motor Vehicle Traffic Crashes as a Leading Cause of Death in the United States, 2002", Traffic Safety Facts, Research note, National Highway Traffic Safety Administration (NHTSA), 2005.

[28] M. Treiber, D. Helbing, "Memory Effects in Microscopic Traffic Models and Wide Scattering in Flow-Density Data," Physical Review E, 68, 46119/1-46119/8, 2003.

[29] M. Treiber, A. Kesting, D. Helbing, "Delays, Inaccuracies and Anticipation in Microscopic Traffic Models", Physica A, 360(1), 71-88, doi:10.1016/j.physa.2005.05.001, 2006.

[30] T.J. Triggs, "Driver brake reaction times: Unobtrusive measurement on public roads", Public Health Review, 15, 275-290, 1987.

[31] J. Zhang, P. Ioannou, "Control of Heavy-Duty Trucks: Environmental and Fuel Economy Considerations," University of Southern California, Los Angeles TO4203, 2004. 15, 275290, 1987. 